\title{
Inheritance of RAPD fragments in haploid and diploid tissues of Pinus sy/vestris (L.)
}

\author{
MENG-ZHU LU, ALFRED E. SZMIDT* \& XIAO-RU WANG \\ Department of Forest Genetics and Plant Physiology, Swedish University of Agricultural Sciences, S-901 83 Umeå, \\ Sweden
}

\begin{abstract}
We studied the inheritance of RAPD fragments in haploid and diploid tissues of eight parents and 80 diploid controlled $\mathrm{F}_{1}$ progeny of Pinus sylvestris (L.). Most fragments detected in the diploid tissue of parents were also present in their haploid macrogametophytes. All fragments observed in the parents were found in the progeny. Individual fragments amplified by a single primer were observed in unrelated trees suggesting that primer competition is not likely to cause 'epistatic' effects on RAPD polymorphism. Segregation of all variable fragments observed in haploid and diploid materials fitted the proportions expected for a dominant Mendelian trait. The segregating fragments were either present or absent confirming the dominant character of RAPD variation reported in most previous studies. None of the fragments detected in this study appeared to originate from uniparentally inherited cytoplasmic DNA. Our present results provide additional support for the usefulness of RAPD fragments as genetic markers. Nevertheless, the few differences observed between haploid and diploid tissues with respect to some fragments emphasize the need for inheritance studies preceding the use of RAPD fragments as genetic markers.
\end{abstract}

Keywords: controlled cross, diploid bud, haploid macrogametophyte, inheritance, Pinus sylvestris, RAPDs.

\section{Introduction}

Random amplified polymorphic DNA (RAPD) markers have become popular tools in genetic research. The technique is based on polymerase chain reaction using short arbitrary primers to amplify at random DNA products from the genomic fragments being paired by the primers (Williams et al., 1990). By using commercially available primers it is possible to obtain a large number of independent genetic markers which apparently make this method particularly attractive for genetic studies. Furthermore, the simplicity and rapidity of RAPD analysis, as well as the very small amount of DNA needed, appears to favour it over the more laborious RFLP technique (Williams et al., 1990).

Despite the continuously increasing number of studies employing RAPDs, relatively little is known about the genetics of this new category of markers. Conifers are particularly well suited for studies on RAPD inheritance because they have both haploid and

\footnotetext{
${ }^{*}$ Correspondence.
}

diploid tissues (Bartels, 1971). So far, however, this advantage has not been fully utilized and only one type of tissue (haploid or diploid) was used in the earlier studies (Carlson et al., 1991; Tulsieram et al., 1992; Kaya \& Neale, 1993; Nelson et al., 1993). As a consequence, nothing is known about the concordance between RAPD fragments amplified from haploid and diploid tissues of the same individual. Similarly, there is no information about the genomic origin of RAPDs in conifers. Clearly, more studies addressing the genetics of RAPDs are needed to assess fully the potential usefulness of this new source of markers in the genetic analysis of conifers.

Here, we report results from a study on the inheritance of RAPD fragments in haploid and diploid tissues of unrelated individuals of Pinus sylvestris (L.) and their $F_{1}$ progeny from a partial diallel cross. We ask the following questions: (i) do RAPD fragments amplified from haploid and diploid tissues of the same individual show the same patterns? (ii) are some RAPD fragments amplified from uniparentally inherited organellar genomes? and (iii) do RAPD fragments segregate as dominant Mendelian traits? 


\section{Materials and methods}

\section{Plant material}

Material for this study came from eight 'plus trees' of $P$. sylvestris, originating from different parts of northern Sweden, and from a partial diallel cross among these parents (Table 1). Detailed information about this cross has been presented elsewhere (Lindgren \& Wang, 1986). Two types of samples were collected from the parental trees. The first type of sample was open-pollinated half-sib seed collected individually from each parent. The second type of sample represented dormant buds taken from several different parts of the crown of each parent. The controlled $F_{1}$ progeny material included four different crosses and their reciprocals (Table 1). Dormant buds were collected from ten $F_{1}$ individuals in each cross. All materials were stored at $-20^{\circ} \mathrm{C}$ until DNA extraction.

\section{DNA extraction and amplification}

Haploid macrogametophytes were separated individually from half-sib seed collections. Total DNA from diploid buds and individual haploid macrogametophytes was extracted as described by Doyle \& Doyle (1990) and suspended in TE buffer (0.1 mM EDTA). The optimal reaction for RAPD analysis was set up at the following conditions: $1 \times$ reaction buffer, $0.5 \mathrm{U}$ Taq DNA polymerase (Pharmacia), $0.3 \mu \mathrm{M}$ of the 10 -mer random primer, $150 \mu \mathrm{M}$ dNTPs and 25-50 ng template DNA for a total volume of $25 \mu \mathrm{L}$. The amplification conditions were as follows: the first step of 3 min at $94^{\circ} \mathrm{C}$, followed by 40 cycles of $1 \mathrm{~min}$ at $94^{\circ} \mathrm{C}, 2$ min at $36^{\circ} \mathrm{C}$ and $2 \mathrm{~min}$ at $72^{\circ} \mathrm{C}$. The final step was 10 min at $72^{\circ} \mathrm{C}$. Amplification was carried out using a programmable thermocycler PTC100 (MJ Research). The arbitrary primer kits ' $A$ ' and ' $\mathrm{X}$ ' were purchased from Operon Technologies (Alameda, CA, USA). A total of 30 primers (OPA01 to 20 and OPX01 to 10) was screened in this study.

Table 1 List of controlled crosses used for analysis of RAPD variation

\begin{tabular}{cccc}
\hline Cross no. & \& Parent & & ơ Parent \\
\hline 1 & AC1005 & $\times$ & AC1019 \\
2 & AC1019 & $\times$ & AC1005 \\
3 & AC1014 & $\times$ & BD1178 \\
4 & BD1178 & $\times$ & AC1014 \\
5 & AC1016 & $\times$ & AC4210 \\
6 & AC4210 & $\times$ & AC1016 \\
7 & BD1032 & $\times$ & Y3020 \\
8 & Y3020 & $\times$ & BD1032 \\
\hline
\end{tabular}

The amplification products were separated in 1.5 per cent agarose gels (FMC) in $0.5 \times \mathrm{TBE}$ buffer. The banding patterns were visualized under UV light and photographed using a Polaroid camera. The DNA standard was a 1 kilobase ladder (BRL). The sizes of the amplified fragments were estimated using the algorithm developed by Schaffer \& Sederoff (1981). The reproducibility of the amplification products was tested at least twice for each experiment.

\section{Data scoring and analysis}

Amplified fragments, named by the primer used and the molecular weight in base pairs (bp), were scored as the presence $(+$ or $A$ ) or absence $(-$ or $a)$ of a fragment as described by Echt et al. (1992); namely, a fragment was counted only if the phenotypic classes were consistent and clearly distinguishable. In instances where two or more phenotypic classes were classified as fragment intensity differences, the fragment was counted only if one of the classes was its virtual absence, and the intensity variants were summed into a single 'presence' class. Only fragments that showed polymorphism in at least one parent analysed in this study were considered. Fragments showing only intensity variation were omitted. Similarly, diffuse and/or very weak fragments were not scored. Such fragments have been reported to possess the greatest propensity for poor reproducibility (Heun \& Helentjaris, 1993). Fragments were scored by three persons independently and then compared.

Forty macrogametophytes from parent BD1032 were used to study the segregation of RAPD fragments in haploid macrogametophyte tissue. To study inheritance of these fragments in the diploid $F_{1}$ progeny, ten individuals were analysed for each cross. The agreement of the observed segregation ratios of RAPD fragments with those expected for a dominant Mendelian trait was calculated using $\chi^{2}$-tests. To decide the zygosity of the individual parents, ten haploid macrogametophytes were analysed for each tree. Assuming a Mendelian 1:1 segregation of individual RAPD fragments, this sample size is sufficient for detection of heterozygotes with 99.8 per cent probability (Morris \& Spieth, 1978).

\section{Results and discussion}

\section{Primer screening}

In this study we tried to assess several genetic properties of RAPD fragments that are relevant to their use in the genetic analysis of forest conifers. To identify primers that detect polymorphism, 30 primers were screened using DNA from diploid buds from the eight 
parents. Of these 30 random primers, nine primers (OPA04, OPA06, OPA12, OPA13, OPA20, OPX04, OPX05, OPX08 and OPX10) failed to yield amplification products. The remaining 21 primers yielded a total of 137 reproducible fragments (data not shown). Of these 137 fragments, 95 fragments (69 per cent) were polymorphic among the parents, with an average of 4.52 fragments per primer. The number of amplified fragments varied from three to 13 per primer, and the size of the fragments ranged from 300 to $2000 \mathrm{bp}$. Based on these preliminary results, we have selected six primers $\{$ OPA01, OPA02, OPA05, OPA07, OPA15 and OPA18) which gave the best amplification products and have used them for the analysis of RAPD inheritance in four controlled crosses and their reciprocals. Four of these primers (OPA02, OPA05, OPA07 and OPA18) were used to study RAPD inheritance in haploid macrogametophytes from the BD1032 parent.

\section{Genomic origin of RAPD fragments}

The present analysis of reciprocal crosses made possible the detection of uniparentally inherited fragments. In conifers, chloroplast and mitochondrial DNAs are inherited from only paternal and maternal parents respectively (e.g. Szmidt et al., 1987; Neale \& Sederoff, 1989). Therefore, fragments amplified from these two genomes would appear only in one type of cross. We never detected a single instance of a reproducible uniparentally inherited fragment such as those reported by Kazan et al. (1993). This agrees with the results obtained by Heun \& Helentjaris (1993), who also did not detect such fragments in Zea mays. Therefore, for further analysis, the progeny of individual crosses and their corresponding reciprocals were pooled.

The lack of cytoplasmic fragments in amplification products derived from total DNA extracts could result from a low contribution of chloroplast or mitochondrial DNA to the total DNA used as a substrate (Williams et al., 1990; Heun \& Helentjaris, 1993). Alternatively, there may be fewer cases of the sequences necessary for single primer amplifications in these genomes than in the nuclear genomes (Heun \& Helentjaris, 1993). Our preliminary data on the RAPD variation in the purified chloroplast DNA extracts from $P$. sylvestris show, however, that the primers used in this study can produce chloroplast DNA-specific fragments (X.-R. Wang, unpublished data). It appears therefore that the lack of cytoplasmic RAPD fragments in the total DNA extracts observed in this study was the result of primer competition rather than the lack of amplifiable sequences in the cytoplasmic DNAs. This particular finding is somewhat disappointing because polymorphic cytoplasmic markers are indispensable in studies of introgression and gene flow in conifers (e.g. Dong \& Wagner, 1994; Ennos, 1994; Wang \& Szmidt, 1994). To overcome this shortcoming we are currently studying polymorphism revealed by primers that are specific for uniparentally inherited DNAs such as those reported by, for example, Taberlet et al. (1991) and Suzuki et al.(1993).

\section{Inheritance of RAPD fragments in haploid macrogametophytes}

The results of RAPD fragment segregation in the haploid macrogametophytes from parent BD1032 are presented in Table 2. The four primers used in the present analysis revealed 15 fragments that showed presence/absence polymorphism and conformed to the 1:1 ratio expected for a Mendelian trait (Fig. 1, Table 2). None of these fragments segregated in a codominant fashion, i.e. they always showed only one of two possible size variants. Our present results corroborate previous reports where a generally good fit to Mendelian expectations and dominant behaviour of RAPD variation has been observed (Tulsieram et al., 1992; Bucci \& Menozzi, 1993; Nelson et al., 1993).

\section{Inheritance of RAPD fragments in diploid progeny}

To our knowledge, published data on RAPD inheritance based on the analysis of controlled crosses is available for only two conifers: Picea glauca and Pseudotsuga menziesii (Carlson et al., 1991). The results from the present analysis of RAPD variation in parents and pooled progeny samples are summarized in Table 3. The fragment pattern revealed by the OPA15 primer in the cross $\mathrm{AC} 1005 \times \mathrm{AC} 1019$ is presented in Fig. 2. A total of 32 different fragments was observed. Three different situations were studied with respect to the occurrence of individual RAPD fragments in parents: (i) both parents shared the fragment; (ii) the fragment was present in one parent but absent in the other; and (iii) both parents lacked the fragment. The third situation could be studied only for fragments observed in at least one of the six remaining parents. In all three situations a dominant character of the RAPD fragment variation was assumed.

The first situation involves one of the following three hypothetical combinations of the parental genotypes: $A A \times A A, A A \times A a$ or $A a \times A a$. The first two combinations involving a dominant homozygote $(A A)$ were expected to yield monomorphic progeny that shared the parental fragment. In the cross between two dominant heterozygotes $(A a)$, the fragment was expected to segregate 3:1 among the progeny. We observed 17 cases where a fragment was shared by both parents. In nine of these 17 cases, the fragment 
occurred in all of the progeny, and it was not possible to decide the parental genotypes. These ambiguous genotypes were denoted as $A_{-}$- (Table 3 ). In the remaining eight cases, a fragment was present in both parents but showed presence/absence polymorphism in the progeny. In all these eight cases, the segregation of the fragment among the progeny did not deviate significantly $\left(P \geq 0.05, \chi^{2}\right.$-test $)$ from the $3: 1$ ratio expected for a dominant Mendelian trait (Table 3).

The second situation can occur when one parent possessing a fragment is either a dominant homozygote $(A A)$ or a dominant heterozygote $(A a)$ and the other a recessive homozygote $(a a)$. In the former case $(A A \times a a)$, all progeny were expected to possess the fragment found in one of the parents. In the latter case

Table 2 Segregation of RAPD fragments in macrogametophytes from the parent BD1032 and $\chi^{2}$ analysis for goodness of fit to a 1:1 ratio

\begin{tabular}{lcccccccc}
\hline & \multicolumn{2}{c}{ Observed } & \multicolumn{2}{c}{ Expected } & & & \\
\cline { 2 - 3 } Fragment & + & - & + & - & $n$ & $\chi^{2}$ & $P$ value \\
\hline OPA02-700 & 23 & 14 & 18.5 & 18.5 & 37 & 2.19 & $>0.10$ \\
OPA02-550 & 19 & 18 & & 18.5 & 18.5 & 37 & 0.03 & $>0.80$ \\
OPA05-1650 & 17 & 17 & 17.0 & 17.0 & 34 & 0.00 & $>0.90$ \\
OPA05-730 & 17 & 17 & 17.0 & 17.0 & 34 & 0.00 & $>0.90$ \\
OPA05-700 & 20 & 14 & 17.0 & 17.0 & 34 & 1.06 & $>0.30$ \\
OPA05-600 & 17 & 17 & 17.0 & 17.0 & 34 & 0.00 & $>0.90$ \\
OPA07-1800 & 14 & 13 & 13.5 & 13.5 & 27 & 0.04 & $>0.80$ \\
OPA07-1400 & 15 & 12 & 13.5 & 13.5 & 27 & 0.33 & $>0.50$ \\
OPA07-1300 & 17 & 10 & 13.5 & 13.5 & 27 & 1.81 & $>0.10$ \\
OPA07-650 & 15 & 12 & 13.5 & 13.5 & 27 & 0.33 & $>0.50$ \\
OPA07-300 & 14 & 13 & 13.5 & 13.5 & 27 & 0.04 & $>0.80$ \\
OPA18-850 & 15 & 20 & 17.5 & 17.5 & 35 & 0.71 & $>0.30$ \\
OPA18-800 & 20 & 15 & 17.5 & 17.5 & 35 & 0.71 & $>0.30$ \\
OPA18-650 & 18 & 17 & 17.5 & 17.5 & 35 & 0.03 & $>0.80$ \\
OPA18-450 & 22 & 13 & 17.5 & 17.5 & 35 & 2.31 & $>0.10$ \\
\hline
\end{tabular}

+ : fragment present; - : fragment absent; $n$ : sample size.
$(A a \times a a)$, the fragment was expected to show presence/absence polymorphism, and to segregate in a 1:1 ratio among the progeny. We found 14 cases where a fragment was present in one parent but absent in the other. In three of these 14 cases, the fragment occurred in all full-sib progeny. In the remaining 11 cases, the fragment present in one of the parents showed presence/absence polymorphism in the progeny that did not deviate significantly $\left(P \geq 0.05, \chi^{2}\right.$-test $)$ from the 1:1 ratio expected for a dominant Mendelian trait (Table 3).

Most earlier studies concerned with RAPD inheritance in controlled crosses reported only results for parents and progenies that showed the presence of a fragment. However, assuming a truly dominant character of the RAPD polymorphism it appears necessary to test cases where both parents show fragment absence, i.e. are recessive homozygotes. We think that analysis of this combination of the parental genotypes also provides relevant information about the genetic behaviour of RAPDs. The situation where a fragment was absent from both parents could be studied for the following five fragments: OPA01-1000, OPA02-700, OPA02550, OPA05-1650 and OPA05-730. These fragments were found in parents BD1032 and AC1019 (Tables 2 and 3). As expected for a cross between two recessive homozygotes, all these five fragments were also absent from the progeny (Table 3). This finding reaffirms dominant, Mendelian behaviour of RAPD fragments observed in this and other studies.

In summary, the segregation of all of the variable fragments found in this material was consistent with a biparental diploid mode of inheritance expected for a dominant trait. A similar mode of inheritance of RAPD fragments has been reported for most other organisms (Carlson et al., 1991; Hunt \& Page, 1992; Roy et al., 1992; Heun \& Helentjaris, 1993; Kazan et al., 1993; Quiros et al., 1993; Rothuizen \& van Wolferen, 1994; Rowland \& Levi, 1994).
Fig. 1 RAPD fragments amplified by the OPA1 8 primer in haploid macrogametophytes of parent BD1032. $\mathrm{S}: 1 \mathrm{~kb}$ ladder.

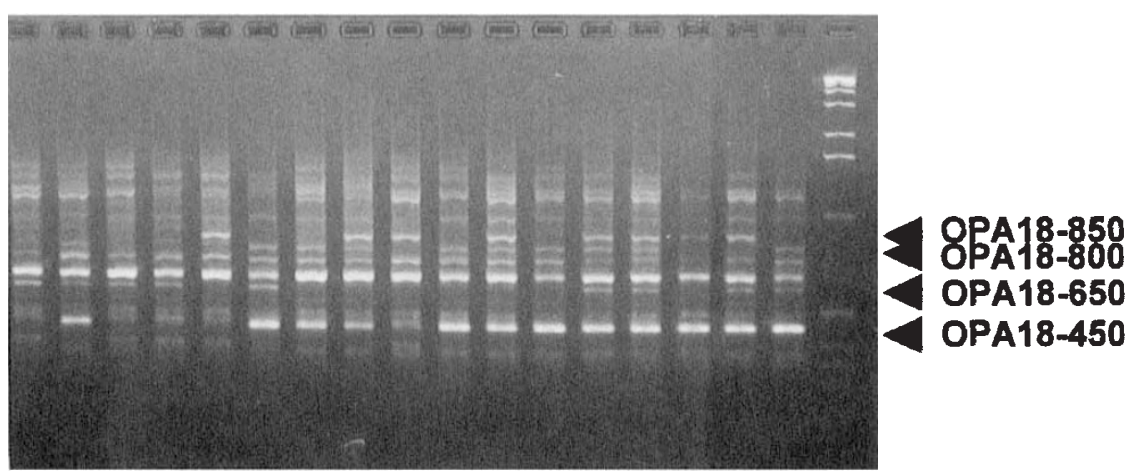






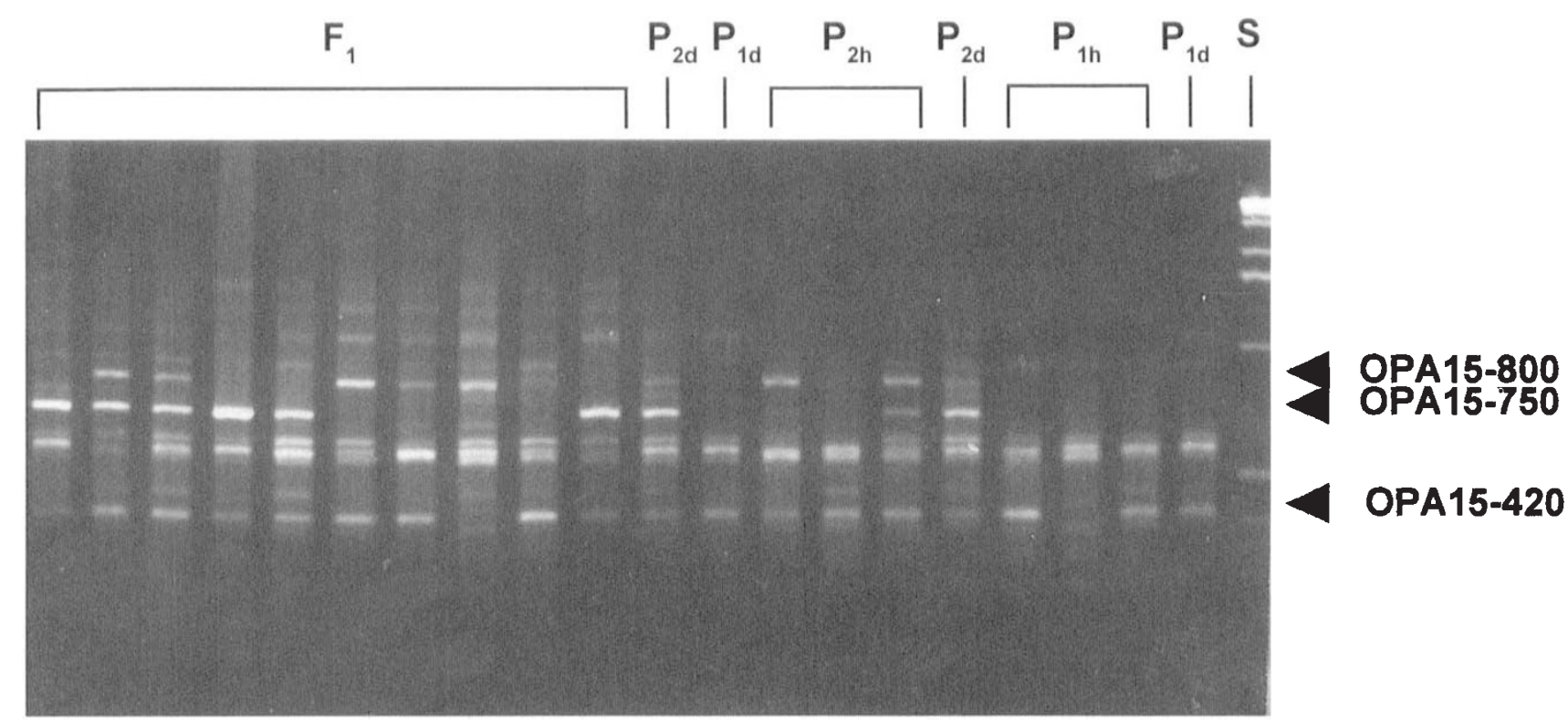

Fig. 2 RAPD fragments amplified by the primer OPA15 in parents and progeny from the cross AC1005 $\times$ AC1019.

$F_{1}$ : progeny; $P_{1 h}: A C 1005$ haploid macrogametophyte; $P_{1 d}: A C 1005$ diploid bud; $P_{2 h}$ : AC1019 haploid macrogametophyte; $\mathrm{P}_{2 \mathrm{~d}}$ : AC1019 diploid bud;

$\mathrm{S}: 1 \mathrm{~kb}$ ladder.

The dominant character of RAPD variation limits the inference of the parental genotype when data are available for only diploid material (Roy et al., 1992), i.e. when a fragment is detected in both parents and in all of the full-sib progeny, it is not possible to distinguish between a dominant homozygote $(A A)$ and a dominant heterozygote $(A a)$ in either of the two parents involved in the cross. This limitation can be alleviated by analysis of haploid tissues of the individual parents. However, to utilize this advantage it is necessary to ascertain that individual RAPD fragments can be detected in both types of tissue. Amplification products are not always concordant between haploid and diploid material. For instance, over 25 per cent of the segregating RAPD fragments were only informative in the haploid drones of Apis mellifera (Hunt \& Page, 1992). Conversely, some other fragments were present in diploid workers and queens but not in the haploid drones. To test whether the ploidy level can affect RAPD pattern in $P$. sylvestris the genotypes of individual parents inferred from analysis of the controlled crosses were compared with the genotypes inferred from analysis of haploid macrogametophytes. Usually, the parental genotypes inferred from diploid material conformed to those inferred from analysis of haploid macrogametophytes. In three cases, however, the parental genotypes inferred from diploid and haploid tissues were different. In two of these three cases, the fragment was present in both parents and segregated 3:1 among the progeny suggesting that these parents are dominant heterozygotes (fragments OPA05-1200 and OPA07-420 in crosses $\mathrm{AC} 1016 \times \mathrm{AC} 4210$ and $\mathrm{Y} 3020 \times \mathrm{BD} 1032$, respectively, marked with $b$ in Table 3). However, the heterozygous character of these parents was not apparent in their haploid macrogametophytes (Table 3). The observed discrepancy could be explained by an undetected heterozygosity of the parents because of the limited number of analysed macrogametophytes. This suggestion should be confirmed by analysis of additional macrogametophytes. The remaining case showed a different kind of discrepancy. The fragment OPA05-1700 (marked with $c$ in Table 3) was observed in diploid tissue of AC4210 but was absent in haploid macrogametophytes of this parent. However, it was present in both haploid and diploid tissues of the other parent AC1016. It therefore appears that the observed discrepancy between haploid and diploid tissues with respect to the OPA05-1700 fragment was not necessarily a result of the difference in the ploidy level. Analysis of additional macrogametophytes and progeny should help to explain the observed discrepancy.

It has been suggested that because of varying number of templates among individual parents the RAPDs can be subject to 'epistatic' effects, i.e. they can be amplified in one genetic background but not in another (Heun \& Helentjaris, 1993). The template homologous to a certain primer may occur in several 
different parents. However, particular parents may differ with respect to the number and homology of other, similar templates, As a result, an individual RAPD fragment could be amplified in only some individuals but would not appear in others because of the primer competition among other more abundant or homologous templates. This possibility can be verified by comparing RAPD variation detected by a particular primer among the progeny from crosses between unrelated parents such as those used in this study. The fragments amplified by the OPA01 primer (marked with asterisk in Table 3) could be studied in two different crosses: BD1178 $\times$ AC1014 and $\mathrm{AC} 1005 \times \mathrm{AC} 1019$. The observed polymorphism of RAPD fragments amplified by OPA01 did not suggest that varying number of templates among the parents causes 'epistatic' effects. For instance, the OPA01-750 fragment was present in all four parents and their progeny. The OPA01-500 fragment segregated in expected proportions in both crosses. In addition, for all fragments amplified by OPA01 the parental genotypes inferred from diploid material conformed to those inferred from haploid macrogametophytes.

\section{Applications of RAPD markers}

Most of the earlier literature on RAPDs has focused on the use of this new category of markers for mapping or genetic diagnostics (e.g. Carlson et al., 1991; Tulsieram et al., 1992; Heun \& Helentjaris, 1993; Nelson et al., 1993; Quiros et al., 1993; Chaparro et al., 1994). We are more interested in the usefulness of these markers for population genetic analysis. The dominant character of RAPD markers found in this and other studies implies that they will be less informative in population analysis than allozymes and RFLPs (Roy et al., 1992). In conifers, this disadvantage can be alleviated by using haploid macrogametophytes of the individuals investigated. However, such an approach requires the analysis of multiple samples per individual, and is limited to only seed-producing individuals. We feel, however, that the limitations of RAPDs because of their dominant character can be compensated by apparently high numbers of easily accessible polymorphic markers which provide insights into many until now unexplored regions of the genome. As demonstrated by recent studies, RAPD markers can provide new valuable information about the structure and variation of populations (e.g. Huff et al., 1993; Liu \& Furnier, 1993; Hilu, 1994). Only eight individuals of $P$. sylvestris and $80 F_{1}$ progeny were analysed in this study. Thus, our present data tell us little about the level of polymorphism of the RAPD markers in this species. However, our recent analysis of RAPD variation in natural populations of $P$. sylvestris indicates that similarly to other species this taxon is characterized by a substantial RAPD polymorphism (A. E. Szmidt, unpublished data). The few discrepancies in RAPD patterns observed between haploid and diploid tissues emphasize that the use of RAPD fragments as genetic markers must be preceded by rigorous inheritance studies. Such studies, employing haploid and diploid tissues, are particularly important in conifers, for which only one type of tissue is usually analysed. In addition, the sequence homology between the fragments of similar size found in this study remains to be verified by hybridization experiments. It is likely, however, that fragments shared by two conspecific individuals are allelic (Kazan et al., 1993).

\section{Acknowledgements}

We thank Dag Lindgren, Swedish University of Agricultural Sciences, Umeå, for help in obtaining plant material for this study. Thanks are also due to Ingabritt Carlsson and Roger Granbom for skilful technical assistance. This study was sponsored with grants from the Swedish Council for Forestry and Agricultural Research (SJFR), the National Research Council (NFR), the Kempe Foundation, and the Nordic Council of Ministers (NKJ).

\section{References}

BARTELS, H. 1971. Genetic control of multiple esterases from needles and macro-gametophytes of Picea abies. Planta, 99, 283-289.

BUCC1, G. AND MENOZZI, P. 1993. Segregation analysis of random amplified polymorphic DNA (RAPD) markers in Picea abies Karst. Mol. Ecol., 2, 227-232.

CARLSON, J. E., TULSIERAM, L. K., GLAUBITZ, J, C., LUK, v. W. K. KAUFFELDT, C. AND RUTLEDGE, R. 1991. Segregation of random amplified DNA markers in $F_{1}$ progeny of conifers. Theor. Appl. Genet., 83, 194-200.

CHAPARRO, J. X., WERNER, D. J., O'MALLEY, D. AND SEDEROFF, R. R. 1994. Targeted mapping and linkage analysis of morphological isozyme and RAPD markers in peach. Theor. Appl. Genet., 87, 805-815.

DONG, J. S. AND WAGNER, D. B. 1994. Paternally inherited chloroplast polymorphism in Pinus: estimation of diversity and population subdivision, and tests of disequilibrium with a maternally inherited mitochondrial polymorphism. Genetics, 136, 1187-1194.

DOYLE, J. J. AND DOYLE, J. L. 1990. Isolation of plant DNA from fresh tissue. Focus, 12, 13-15.

ECHT, C. S., ERDAHL, L. A. AND McCOY, T. J. 1992. Genetic segregation of random amplified polymorphic DNA in diploid cultivated alfalfa. Genome, 35, 84-87. 
ENNOS, R. 1994. Estimating the relative rates of pollen and seed migration among plant populations. Heredity, 72, 250-259.

HEUN, M. AND HELENTJARIS, T. 1993. Inheritance of RAPDs in $\mathrm{F}_{1}$ hybrids of corn. Theor. Appl. Genet., 85, 961-968.

HILU, K. W. 1994. Evidence from RAPD markers in the evolution of Echinochloa millets (Poaceae). Plant Syst. Evol., 189, 247-257.

HUFF, D. R., PEAKALL, R. AND SMOUSE, P. E. 1993. RAPD variation within and among natural populations of outcrossing buffalograss [Buchloe dactyloides (Nutt) Engelm]. Theor. Appl. Genet., 86, 927-934.

HUNT, G. J. AND PAGE, R. E. 1992. Patterns of inheritance with RAPD molecular markers reveal novel types of polymorphism in the honey bee. Theor. Appl. Genet., 85, 15-20.

KAYA, Z. AND NEALE, D. B. 1993. Random amplified polymorphic DNA (RAPD) polymorphisms in Pinus nigra subsp. pallasiana and Pinus brutia. Turkish J. Agric. Forestry, 17, 295-306.

KAZAN, K., MANNERS, J. M. AND CAMERON, D. F. 1993. Inheritance of random amplified polymorphic DNA markers in an interspecific cross in the genus Stylosanthes. Genome, 36, 50-56.

LINDGREN, D. AND WANG, Q.-Y. 1986. Are genetic results influenced by the environment during seed maturation? In: Lindgren, D. (ed.) Joint Meeting of IUFRO WP. on Breeding Theory, Progeny Testing and Seed Orchards, 13-17 October, 1986, Umeå, Sweden, pp. 192-199. Swedish University of Agricultural Sciences.

LIU, z. AND FURNIER, G. R. 1993. Comparison of allozyme, RFLP, and RAPD markers for revealing genetic variation within and between trembling aspen and bigtooth aspen. Theor. Appl. Genet., 87, 97-105.

MORRIS, R. W. AND SPIETH, P. T. 1978. Sampling strategies for using female gametophytes to estimate heterozygosities in conifers. Theor. Appl. Genet., 51, 217-222.

NEALE, D. B. AND SEDEROFF, R. R. 1989. Paternal inheritance of chloroplast DNA and maternal inheritance of mitochondrial DNA in loblolly pine. Theor. Appl. Genet., 77, 212-216.
NELSON, C. D., NANCE, W. L. AND DOUDRICK, R. L. 1993. A partial genetic linkage map of slash pine (Pinus elliottii Engelm. var. elliottii) based on random amplified polymorphic DNAs. Theor. Appl. Genet., 87, 145-151.

QUIROS, C. F., CEADA. A., GEORGESCU, A. AND HU, J. 1993. Use of RAPD markers in potato genetics: segregations in diploid and tetraploid families. Am. Potato J., 70, 35-42.

ROTHUIZEN, J. AND VAN WOLFEREN. M. 1994. Randomly amplified DNA polymorphisms in dogs are reproducible and display Mendelian transmission. Animal Genet., 25, 13-18.

ROWLAND, L. J. AND LEVI, A. 1994. RAPD-based genetic linkage map of blueberry derived from a cross between diploid species (Vaccinium darrowi and V. elliottii). Theor. Appl. Genet., 87, 863-868.

ROY, A., FRASCARIA, N., MACKAY, J. AND BOUSQUET, J. 1992. Segregating random amplified polymorphic DNAs (RAPDs) in Betula alleghaniensis. Theor. Appl. Genet., 85, 173-180.

SCHAFFER, H. E. AND SEDEROFF, R. 1981. Improved estimation of DNA fragment lengths from agarose gels. Analyt. Biochem., 115, 113-122.

SUZUKI, R., KEMP, S. J. AND TEALE, A. J. 1993. Polymerase chain reaction analysis of mitochondrial DNA polymorphism in Ndama and Zebu cattle. Animal Genet., 24, 339-343.

SZMIDT, A. E., ALDEN, T. AND hallgren, J.-E. 1987. Paternal inheritance of chloroplast DNA in Larix. Plant Mol. Biol., 9, 59-64.

TABERLET, P., GIELLY, L., PAUTOU, G. AND BOUVET, J. 1991. Universal primers for amplification of three non-coding regions of chloroplast DNA. Plant Mol. Biol., 17, 1105-1109.

TULSIERAM, L. K., GLAUBITZ, J. C., KISS, G. AND CARLSON, J. E. 1992. Single tree genetic linkage mapping in conifers using haploid DNA from megagametophytes. Bio/Technology, 10, 686-690.

WANG, X.-R. AND SZMIDT, A. E. 1994. Chloroplast DNA variation in a Pinus species complex from Asia. Evolution, 48, 1020-1031.

WILLIAMS, J. G. K., KUBELIK, A. R., LIVAK, K. J., RAFALSKI, J. A. AND TINGEY, S. V. 1990. DNA polymorphisms amplified by arbitrary primers are useful as genetic markers. Nucl. Acids Res., 18, 6531-6535. 